\title{
Cyanures et thiocyanates en toxicologie hospitalière
}

\section{Cyanides and thiocyanates in a hospital toxicology laboratory}

\section{Corinne CHARLIER*(1), Thierry GOUGNARD(1), Denis LAMIABLE ${ }^{(2)}$, Pierre LEVILLAIN ${ }^{(3)}$, Guy PLOMTEUX ${ }^{(1)}$}

(1) Laboratoire de Toxicologie Clinique, CHU de Liège - B-4000 Liège - BELGIQUE (2) Laboratoire de Pharmacologie et Toxicologie, CHRU, Hôpital Maison Blanche F-51092 REIMS Cedex - FRANCE (3) 91250 St Germain les Corbeil - FRANCE

*Auteur à qui adresser la correspondance : Dr Corinne CHARLIER, Laboratoire de Toxicologie Clinique, Tour des Soins Intensifs, $5^{\text {eme }}$ étage, CHU - B35 - B-4000 Liège - BELGIQUE Tél : 003243668818 - Fax : 003243668889 - e-mail : C.Charlier@chu.ulg.ac.be

\section{$R \hat{E} S U M E ́$}

L'ion cyanure est un poison très violent, susceptible d'entrầner chez l'Homme la mort en quelques minutes. Dans l'organisme, sous l'action principale de deux enzymes, la thiosulfate sulfure transférase et la $\beta$ mercaptopyruvate transsulfurase, il se transforme en ion thiocyanate $\left(S C N^{-}\right)$, éliminé par voie urinaire. La détermination du taux sanguin du cyanure est importante pour confirmer le diagnostic d'intoxication. Les différentes méthodes de dosage des cyanures et thiocyanates dans les milieux biologiques sont présentées, en insistant particulièrement sur leur praticabilité et leur sensibilité respectives.

\section{MOTS-CLÉS}

Cyanure, thiocyanate, matrices biologiques, quantification.

\begin{abstract}
SUMMARY
Cyanide is a highly toxic poison, able to very rapidly induce the death. Thiocyanate anion $\left(\mathrm{SCN}^{-}\right)$is a metabolic product of cyanide and cyano-containing organic substances, produced by two major enzymes: thiosulfate sulfure transferase and $\beta$ mercaptopyruvate transsulfurase. Determinations of blood cyanide is important to confirm the diagnostic of intoxication. A survey of methods for the quantitative determination of cyanide and thiocyanate in biological matrices is presented. Particular attention is paid to the rapidity and to the sensitivity of the method.
\end{abstract}

\section{KEY-WORDS}

Cyanide, thiocyanate, biological matrices, quantification. 


\section{Introduction}

L'acide cyanhydrique, encore appelé nitrile formique ou acide prussique, est un liquide très volatil, non inflammable, dont la résorption est extrêmement rapide : quelques secondes par voie pulmonaire et quelques minutes par voie digestive. La résorption par voie percutanée et par voie oculaire est également possible.

L'acide cyanhydrique possède une odeur très caractéristique d'amandes amères qui se perçoit habituellement pour des concentrations dans l'atmosphère de l'ordre de 0,2 à1,0 ppm chez des sujets sains et attentifs. Toutefois, cette odeur n'est pas toujours perçue de la même façon par tout le monde et certaines personnes sont incapables de la détecter même à des concentrations nettement supérieures à $1 \mathrm{ppm}$.

Le risque d'intoxication cyanhydrique aiguë concerne surtout les personnes en contact avec des fumées d'incendie, mais les sources d'intoxication par les cyanures restent diversifiées :

\section{$>$ Fumées d'incendie}

- la combustion des matières organiques ou des polymères de synthèse libère de l'acide cyanhydrique, ce qui explique l'intoxication potentielle des pompiers ou de la population lors de l'inhalation de fumées d'incendie (1). L'acide cyanhydrique peut également être libéré lors de la combustion de substances dites naturelles comme la laine, le bois, le coton, la soie, le caoutchouc, ... Le dégagement de $\mathrm{HCN}$ lors de la combustion thermique est dépendant de la quantité d'azote du matériau et de la température.

\section{> Professionnelles}

- les cyanures alcalins sont employés dans la métallurgie et utilisés dans la fabrication de certaines résines et matières plastiques ;

- l'acide cyanhydrique est également présent dans les gaz de cokerie et de hauts-fourneaux ;

- lors des campagnes de désinfection et surtout de dératisation, l'intoxication cyanhydrique est possible, bien que cet usage soit peu à peu abandonné.

\section{$>$ Accidentelles}

- l'absorption d'une quantité excessive d'eau de lauriercerise (le titre en $\mathrm{HCN}$ total de l'eau de laurier-cerise est de $1 \%$, dont $25 \%$ est sous forme de nitrile mandélique - benzaldéhyde cyanhydrique - et $75 \%$ sous forme libre) peut conduire à une intoxication grave ;

- la cueillette et l'absorption imprudente de substances végétales : baies de sureau noir, groseilles ou champignons (clitocybes), peut avoir de graves conséquences ;

- des intoxications alimentaires sont possibles :

- absorption de viande provenant de bétail contaminé par des semences de lin traitées au cyanure,

- ingestion de haricots exotiques [haricots de Lima (300 $\mathrm{mg}$ de $\mathrm{HCN} / 100 \mathrm{~g}$ ) ou racines de manioc (250 mg $\mathrm{HCN} / 100 \mathrm{~g})]$,

- contamination par du massepain, ou des noyaux de certains fruits (pêches, abricots, prunes) qui contiennent des hétérosides cyanogénétiques (amygdaline) capables de libérer l'acide cyanhydrique par voie enzymatique.

Ces hétérosides peuvent être transformés avec libération de HCN selon le processus schématisé à la Figure 1.

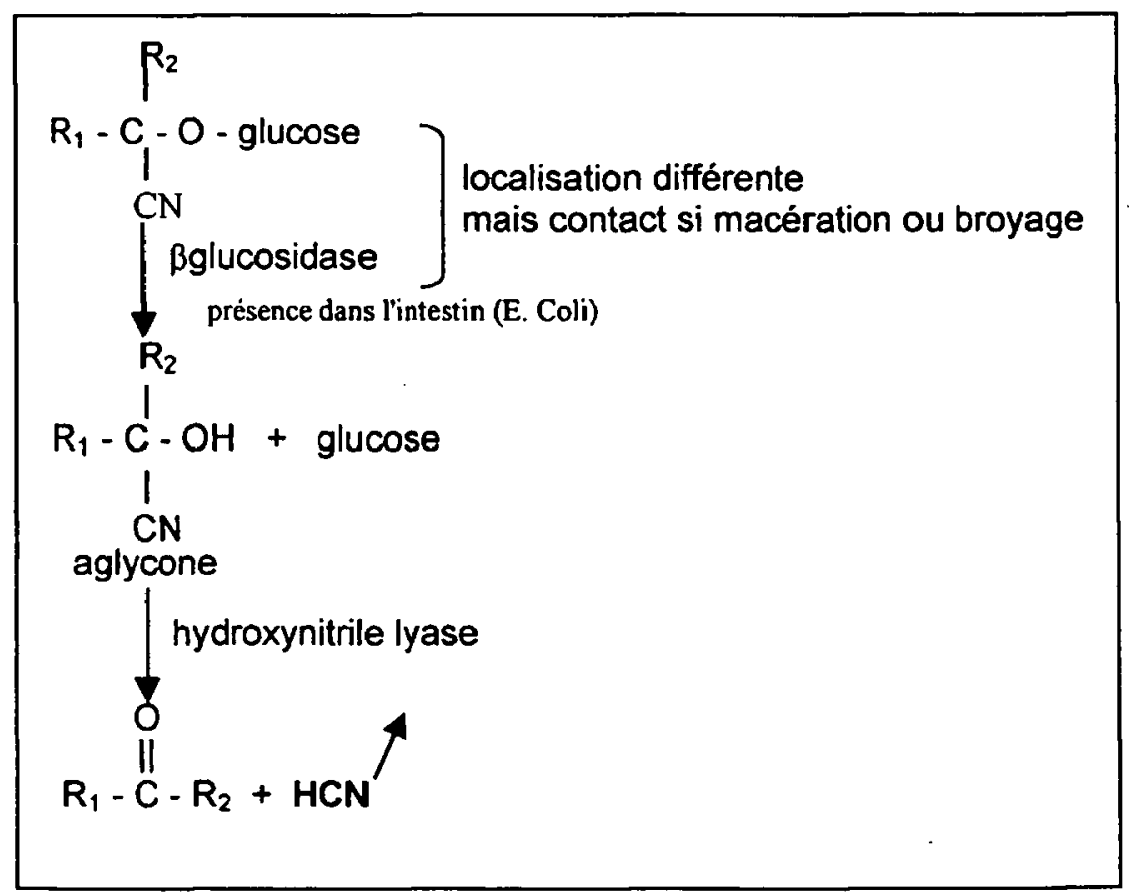

Figure 1 : Libération de $H C N$ à partir des hétérosides cyanogénétiques.

\section{$>$ Fumées de tabac}

- La fumée de tabac est une source non négligeable d'intoxication cyanhydrique, notamment chez les gros fumeurs où la quantité d'acide cyanhydrique inhalée peut être assez importante (la fumée de tabac contient $0,1 \% \mathrm{HCN})$.

D'un point de vue pharmacocinétique, le cyanure est résorbé par ingestion (cyanure métallique), par voie cutanée et par inhalation ( $\mathrm{HCN}$ et nitriles). Le cyanure est distribué dans le sang (98\% dans les globules rouges, $2 \%$ dans le plasma) et dans les tissus (surtout la rate). Il est transformé selon le mécanisme schématisé à la Figure 2. 


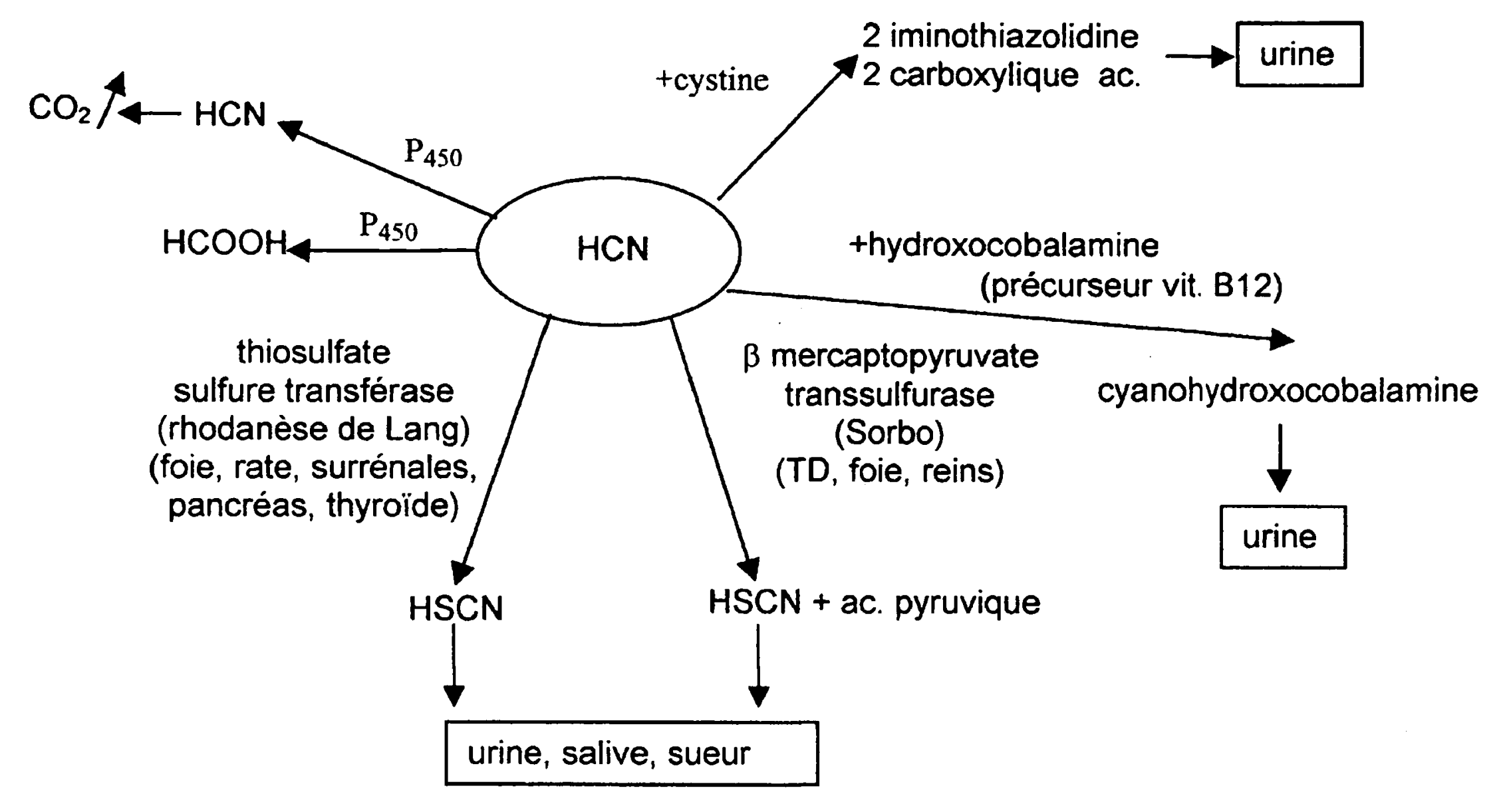

Figure 2 : Métabolisation de l'acide cyanhydrique avec formation de thiocyanate.

L'ion cyanure est un poison cellulaire violent qui inhibe au moins 40 enzymes différents, dont la cytochrome oxydase servant au transport d'électrons dans la chaîne respiratoire pour former les molécules d'ATP, ce qui en fait un enzyme d'importance vitale. Le coenzyme de la cytochrome oxydase, de nature héminique, possède un ion de fer se trouvant à 2 états d'oxydation $(+2$ et +3$)$ permettant ainsi le transport des électrons. En se liant au fer ferrique de la cytochrome oxydase, l'ion cyanure bloque la chaîne respiratoire mitochondriale entraînant un effet anoxiant typique : l'ATP ne peut plus être produit. Le cyanure a le même effet qu'une absence totale d'oxygène. Toutefois, la quantité d'oxygène arrivant au tissu est normale, mais les cellules ne sont plus capables de l'utiliser. Une personne intoxiquée par l'acide cyanhydrique meurt de son incapacité à utiliser l'oxygène.

Les valeurs habituelles des cyanures et thiocyanates dans le sang sont respectivement de $0,02 \mathrm{mg} / \mathrm{l}$ (non fumeurs) à $0,05 \mathrm{mg} / \mathrm{l}$ (fumeurs) pour les cyanures et de moins de $4 \mathrm{mg} / \mathrm{l}$ (non fumeurs) à $20 \mathrm{mg} / \mathrm{l}$ (fumeurs) pour les thiocyanates. Dès $0,65 \mathrm{mg} / \mathrm{l}$ de cyanures, 1 'intoxication est jugée sévère, une concentration de $2,5 \mathrm{mg} / \mathrm{l}$ pouvant entraîner la mort.

Les signes d'intoxication dépendent évidemment du degré d'intoxication :

chronique : le pouls est rapide, quelques vertiges avec bouffées d'angoisse apparaissent, des maux de tête s'installent avec des rougeurs de la peau parfois associés à des vomissements. Dans l'intoxication chronique, un effet toxique secondaire habituel est l'hypothyroïdie, s'expliquant par des propriétés d'inhibition de la captation de l'iodure par la thyroïde due au thiocyanate ;

$>$ aiguë : un tableau clinique associant des atteintes neurologiques (convulsions, coma hypotonique), respiratoire (dépression respiratoire, apnée précédée parfois d'un oedème pulmonaire aigu), cardio-vasculaire (extrasystoles ventriculaires, insuffisance circulatoire) s'installe ;

$>$ mortelle : un coma avec hypotension apparaît. la respiration devient lente et haletante. Une mydriase s'installe. Sans réanimation appropriée, la mort survient rapidement avec arrêt cardiorespiratoire et lésions cérébrales irréversibles.

Cette intoxication présente donc la même physiopathologie qu'un manque complet d'oxygène, avec une diminution de la formation des pyruvates dans le cycle de Krebs et une augmentation des lactates, conduisant à une acidose métabolique.

Deux approches thérapeutiques - symptomatique et spécifique - sont possibles (Figure 3). La correction des symptômes est d'autant plus importante à envisager que l'intoxication est grave, y compris l'oxygénation hyperbare qui permet à l'oxygène de déplacer le cyanure de sa liaison à la cytochrome oxydase. En cas d'intoxica- 


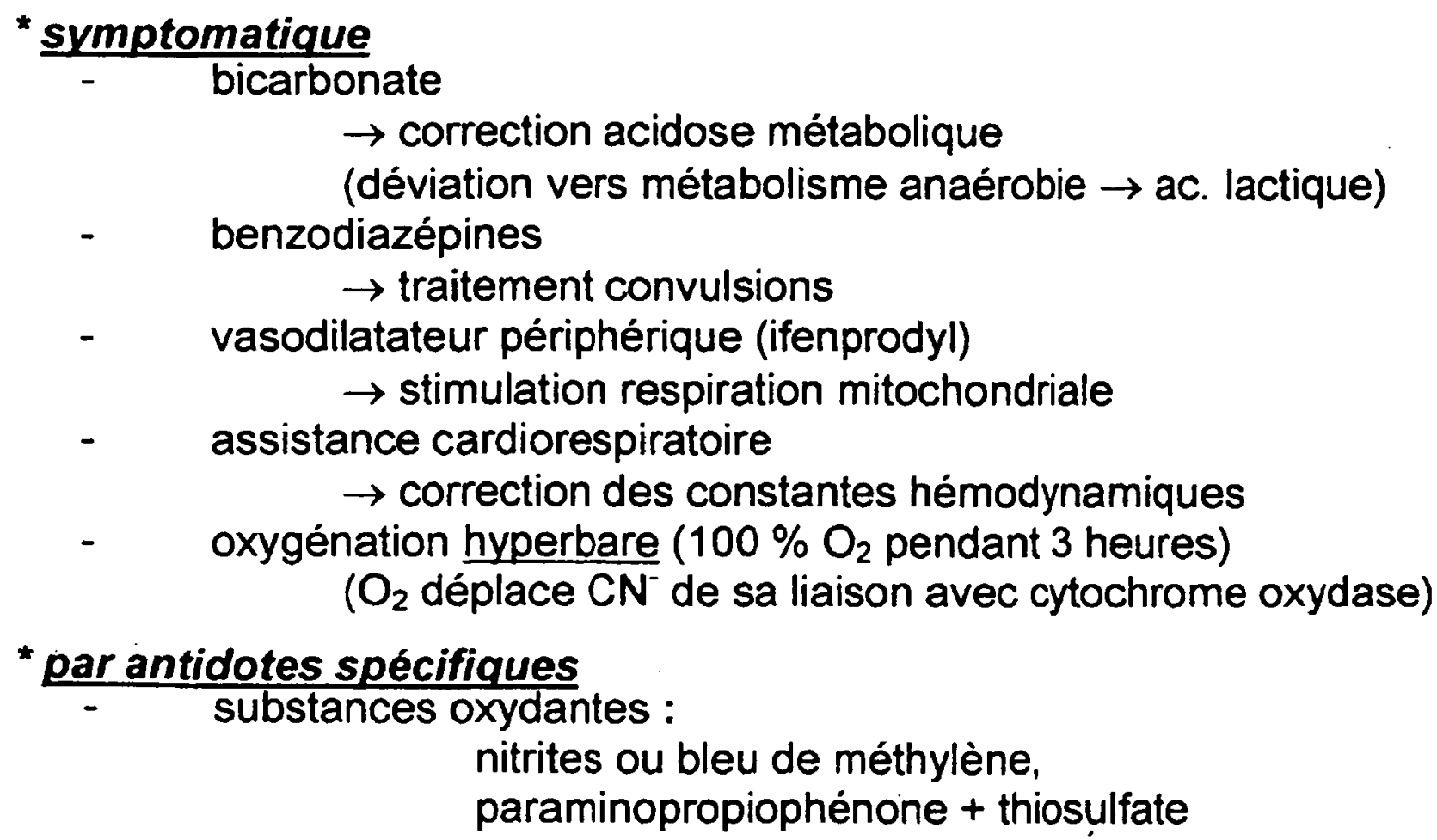

* par antidotes spécifiques

- substances oxydantes :

nitrites ou bleu de méthylène,

paraminopropiophénone + thiosulfate

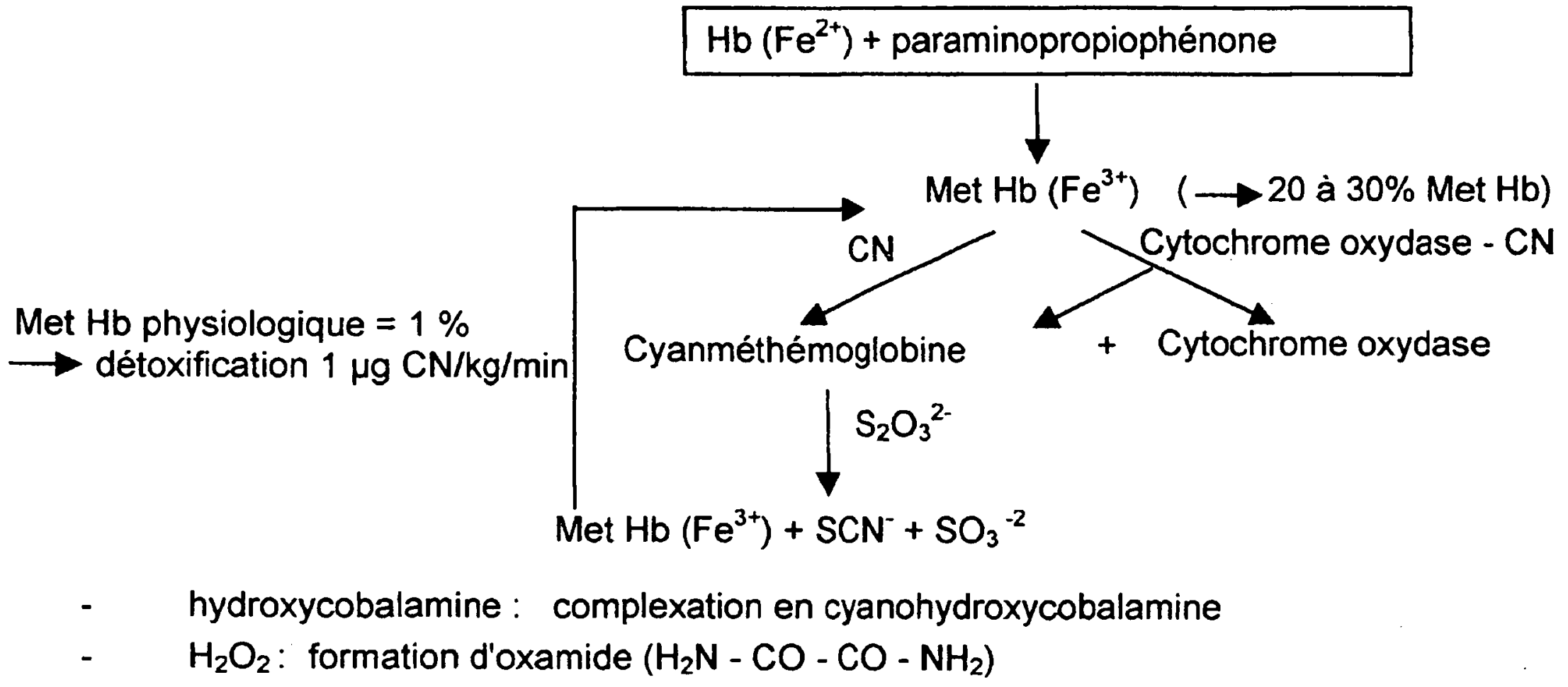

Figure 3 : Traitement de l'intoxication cyanhydrique.

tion massive, il faut un traitement spécifique et capable de fixer les cyanures avec une forte affinité avant qu'ils ne diffusent dans les cellules. Ceci est possible avec l'hydroxycobalamine et des substances oxydantes capables de transformer l'hémoglobine en méthémoglobine (par exemple le mélange paraminopropiophénone-thiosulfate).

L'hydroxycobalamine doit être préférée au traitement oxydant, surtout chez les patients présentant une concentration élevée en carboxyhémoglobine, c'est-àdire chez qui la quantité d'hémoglobine disponible pour fixer l'oxygène est déjà réduite.

La mise en évidence d'une intoxication cyanhydrique se réalisera par:

- le dosage du cyanure dans l'air ambiant par colorimé- trie. La concentration est considérée comme dangereuse au-dessus de 20 ppm et pouvant entraîner la mort audessus de $100 \mathrm{ppm}$

- le dosage du cyanure dans le sang.

La détermination de la concentration des thiocyanates plasmatiques et urinaires peut être utilisée comme témoin de l'intoxication cyanhydrique et non pour évaluer son importance.

\section{Méthodes de dosage}

Plusieurs techniques sont disponibles pour le dosage des cyanures, de la simple diffusion avec quantification par colorimétrie au couplage HS-GC. Le Tableau I résume les principales méthodes adaptées à l'évaluation 
Tableau I : Méthodes de dosage des cyanures dans le sang.

\begin{tabular}{|c|c|c|c|c|c|c|c|}
\hline Technique & $\begin{array}{l}\text { Volume } \\
\text { échantillon }\end{array}$ & Particularités & Délai & Linéarité & Reproductibilité & $\begin{array}{l}\text { Limite de } \\
\text { détection }\end{array}$ & Référence \\
\hline Fluorescence & $200-1000 \mu \mathrm{l}$ & $\begin{array}{l}\text { Cell. Conway détection } \\
\text { modifiée pour éviter } \\
\text { interférence thiosulfate }\end{array}$ & $60 \mathrm{~min}$. & - & - & - & (2) \\
\hline Spectrophotométrie & $200 \mu \mathrm{l}$ & Cell. Conway modifiée & $30 \mathrm{~min}$. & - & - & $0,05 \mathrm{mg} / \mathrm{l}$ & (3) \\
\hline Fluorescence & $1000 \mu \mathrm{l}$ & Cell. Conway à $45^{\circ} \mathrm{C}$ & $\begin{array}{c}30 \mathrm{~min} . \\
\text { (diff. } 20 \mathrm{~min} . \text { ) }\end{array}$ & $0-2,5 \mathrm{mg} / \mathrm{l}$ & $3 \%$ à $1,25 \mathrm{mg} / \mathrm{l}$ & $0,07 \mathrm{mg} / \mathrm{l}$ & (4) \\
\hline Spectrophotométrie & $500 \mu \mathrm{l}$ & $\begin{array}{c}\text { Cellule Conway } \\
\text { (Référence) }\end{array}$ & $\begin{array}{c}130 \mathrm{~min} . \\
\text { (diff. } 120 \mathrm{~min} . \text { ) }\end{array}$ & $0-13 \mathrm{mg} / \mathrm{l}$ & $8 \%$ à $1,25 \mathrm{mg} / \mathrm{l}$ & $28,6.10^{-6} \mathrm{mg}$ & $(5)$ \\
\hline HeadSpace/GC & $200 \mu \mathrm{l}$ & NPD & $35 \mathrm{~min}$. & $0,026-7,8 \mathrm{mg} / \mathrm{l}$ & $5 \%$ à $0,2 \mathrm{mg} / \mathrm{l}$ & $0,039.10^{6} \mathrm{mg}$ & (5) \\
\hline Fluorescence & $20 \mu 1$ & Méthode directe & $45 \mathrm{~min}$. & $2,6-156 \mathrm{mg} / \mathrm{l}$ & - & $2,6.10^{-6} \mathrm{mg}$ & (6) \\
\hline Fluorescence & $100 \mu \mathrm{l}$ & $\begin{array}{l}\text { Dérivation par 2,3- } \\
\text { naphtalène di-aldéhyde } \\
\text { + taurine }\end{array}$ & $45 \mathrm{~min}$. & $0,1 \cdot 10^{-3}-0,2 \mathrm{mg} / \mathrm{l}$ & - & $0,1 \cdot 10^{-3} \mathrm{mg} / \mathrm{l}$ & $(7,8)$ \\
\hline
\end{tabular}

des cyanures, de la simple diffusion avec quantification par colorimétrie au couplage HS-GC. Le Tableau I résume les principales méthodes adaptées à l'évaluation des cyanures dans le sang, avec une sensibilité suffisante pour dépister des intoxications chroniques.

La première méthode proposée repose sur la diffusion dans une cellule de Conway (2). Le même principe est à la base des techniques publiées en 1990 (3), en 1994 (4) et 1995 (5) qui diffèrent essentiellement par le délai de réalisation. Une méthode directe a été décrite par Suzuki (6). Si la méthode la plus sensible semble être le couplage HS-GC-NPD (5), la technique la plus simple est celle qui consiste à doser en fluorescence le complexe formé après dérivation par la 2,3-naphtalènedialdéhyde en présence de taurine $(7,8)$. Cette méthode est également rapide (45 min.) et sensible, puisqu'elle permet de détecter jusqu'à $0,1.10^{-3} \mathrm{mg} / 1$ de cyanure. En ce qui concerne le dosage des thiocyanates plasmatiques et salivaires, les différentes méthodes sont indiquées dans le Tableau II. L'interférence des cyanures et des nitrites est évitée et les limites de détection sont satisfaisantes. La technique qui associe au mieux rapidité, sensibilité et spécificité est celle décrite par Chinaka et al (8), qui permet de quantifier, sur un même échantillon biologique, les cyanures et les thio-

Tableau II : Méthodes de dosage des thiocyanates.

\begin{tabular}{|c|c|c|c|c|c|c|c|}
\hline Technique & $\begin{array}{l}\text { Volume } \\
\text { echantillon } \\
\text { ex }\end{array}$ & Particularites & Délai & Linearité & Reproductibilité & $\begin{array}{l}\text { Limite de } \\
\text { détection }\end{array}$ & Reférence \\
\hline $\begin{array}{l}\text { Spectrophotométrie } \\
\text { (sur COBAS ou } \\
\text { en manuel) }\end{array}$ & $\begin{array}{c}5 \mathrm{ml} \text { salive ou } \\
\text { plasma }\end{array}$ & Sans déprotéiniser & $30 \mathrm{~min}$. & $0-290 \mathrm{mg} / \mathrm{l}$ & $3-8 \%$ & - & (9) \\
\hline HPLC/Fluorescence & $\begin{array}{c}100 \mu \text { l salive ou } \\
\text { plasma }\end{array}$ & $\begin{array}{l}\text { Pas d'interférence } \\
\mathrm{CN} \text { et } \mathrm{NO}^{-}\end{array}$ & $90 \mathrm{~min}$. & $2,9 \cdot 10^{-3}-0,58 \mathrm{mg} / \mathrm{l}$ & $1-2 \%$ & $2,9.10^{-3} \mathrm{mg} / \mathrm{l}$ & (10) \\
\hline HPLC/Fluorescence & $5 \mathrm{ml}$ salive & $\begin{array}{l}\text { Pas d'interférence } \\
\mathrm{CN} \text { ' et } \mathrm{NO} 2\end{array}$ & $60-90 \mathrm{~min}$. & $5,8 \cdot 10^{-3}-5,8 \mathrm{mg} / 1$ & $3-4 \%$ & $5,8 \cdot 10^{-3} \mathrm{mg} / \mathrm{l}$ & (11) \\
\hline HPLC/UV & $100 \mu \mathrm{l}$ sang & $\begin{array}{c}\text { Dérivation par 2,3- } \\
\text { naphtalène di-aldéhyde } \\
+ \text { t taurine }\end{array}$ & $45 \mathrm{~min}$. & $9,86.10^{-3}-10 \mathrm{mg} / \mathrm{l}$ & - & $9,86.10^{-3} \mathrm{mg} / \mathrm{l}$ & (8) \\
\hline
\end{tabular}




\section{Conclusion}

L'ion cyanure est un toxique cellulaire non spécifique d'organe. Le blocage du métabolisme cellulaire aérobie provoque une anoxie tissulaire aiguë avec déviation métabolique vers la voie anaéobie et accumulation d'acide lactique. La détermination de la concentration sanguine de cyanure est importante pour confirmer le diagnostic de l'intoxication. Le dosage des thiocyanates est également utile pour apprécier l'intoxication cyanhydrique ou pour vérifier s'il n'y a pas d'accumulation de ce métabolite dans l'organisme (12). Les méthodes de dosage décrites ici concernent le sang, le plasma ou la salive. Le couplage HS-GC-NPD donne les meilleurs résultats au plan de la sensibilité, mais n'est peut-être pas facilement accessible à tous les laboratoires d'urgence. Un excellent compromis, qui permet un dosage simultané des cyanures et des thiocyanates, est proposé par la méthode chromatographique décrite par Chinaka et al (8), avec détection fluorimétrique des cyanures dérivés et détection U.V. des thiocyanates. Rapide, cette méthode est linéaire de $0,1 \cdot 10^{-3}$ à $0,2 \mathrm{mg} / 1$ de cyanure et de $9,86.10^{-3}$ à $10 \mathrm{mg} / \mathrm{l}$ de thiocyanates. Les limites de détection sont respectivement de $0,1 \cdot 10^{-3} \mathrm{mg} / \mathrm{l}$ et de $9,86 \cdot 10^{-3} \mathrm{mg} / \mathrm{l}$.

\section{Références}

1. Baud F.J., Barriot P., Toffis V., Riou B., Vicaut E., Lecarpentier Y., Bourdon R., Astier A., Bismuth C. Elevated blood cyanide concentrations in victims of smoke inhalation. N. E. J. M. 1991 ; 325 : 1761-1766.

2. Morgan R.L., Way J.L. Fluorimetric determination of cyanide in biological fluids with pyridoxal. J. Anal. Toxicol. $1980 ; 4: 78-81$.

3. Dunn W.A., Siek T.Z. A rapid, sensitive, and specific screening technique for the determination of cyanide. $J$. Anal. Toxicol. $1990 ; 14: 256$.

4. Laforge M., Buneaux F., Houeto P., Bourgeois F., Bourdon R., Levillain P. A rapid spectrophotometric blood cyanide determination applicable to emergency toxicology. J. Anal. Toxicol. 1994 ; 18 : 173-175.

5. Cardeal Z.L., Gallet J.P., Astier A., Pradeau D. Cyanide assay : statistical comparison of a new gas chromatographic calibration method versus the classical spectrophotometric method. J. Anal. Toxicol. $1995 ; 19: 31-34$.

6. Suzuki O., Hattori H., Oya M., Katsumata Y. Direct fluorometric determination of cyanide in human materials. For. Sc. Int. $1982 ; 19: 189-195$.

7. Sano A., Takimoto N., Takitani S. High-performance liquid chromatographic determination of cyanide in human red blood cells by pre-column fluorescence derivatization. J. Chrom. $1992 ; 582$ : 131-135.
8. Chinaka S., Takayama N., Michigami Y, Veda K. Simultaneous determination of cyanide and thiocyanate in blood by ion chromatography with fluorescence and ultraviolet detection. J. Chrom. B., 1998 ; 713 : 353-359.

9. Degiampietro P., Peheim E., Drew D., Graf H., Colombo J.P. Determination of thiocyanate in plasma and saliva without deproteinisation and its validation as a smoking parameter. J. Clin. Chem. Clin. Biochem., 1987 ; 25 : 711-717.

10.Chen S-H., Yand Z-Y., Wu H.L., Kou H-S., Lin S-J. Determination of thiocyanate anion by high-performance liquid-chromatography with fluorimetic detection. J. Anal. Toxicol., $1996 ; 20: 38-42$.

11. Tanabe S., Kitahara M., Nawata M., Kawanabe K. Determination of oxidizable inorganic anions by highperformance liquid chromatography with fluorescence detection and application to the determination of salivary nitrite and thiocyanate and serum thiocyanate. J. Chrom. 1988 ; 424 : 29-38.

12.Dehon B., Lhermitte M. Cyanures et anions. In : Kintz P., ed. Toxicologie et pharmacologie médico-légales. Elsevier, 1998 ; 147-187. 